\title{
Quantifying mechanical ventilation performance: the connection between transport equations and Markov matrices
}

\author{
Anthony D. Fontanini ${ }^{1}$, Umesh Vaidya ${ }^{2}$, Alberto Passalacqua ${ }^{1}$, and Baskar \\ Ganapathysubramanian*1 \\ ${ }^{1}$ Department of Mechanical Engineering, 2100 Black Engineering, Iowa State \\ University, Ames, IA 50010, USA \\ ${ }^{2}$ Dpartment of Electrical and Computer Engineering, 2215 Coover, Iowa \\ State University, Ames, IA 50010, USA
}

\begin{abstract}
Most people spend approximately $90 \%$ of their lives indoors. Thus, designing effective ventilation systems is essential to mitigating problems with indoor air quality. The measures of mechanical ventilation design performance considered in this study are age of air, air residual life time, air residence time, and ventilation effectiveness. This paper presents two different methods to help quantify these measures. The first method is based on transport equations, where a continuous representation of these quantities are calculated. The second method is based on Markov matrices, where a discrete representation of these quantities are calculated. We show 1) how both the continuous and discrete methods are related and 2) that the age of air and residual life time are adjoints. A new transport equation for residual life time along with methods for these quantities using Markov matrices are established. The two approaches are validated and compared using previously established experimental data. The results show that both approaches provide similar results. Using these techniques allows for the quantities of residual life time and residence time to be integrated into the design processes. This paper provides a simple framework that enables designers to get a comprehensive picture of the ventilation systems they design.
\end{abstract}

\section{Introduction}

As people in North America and Europe spend 90\% of their lives indoors [1], [2], the quality of the air they breath is essential to their health. Symptoms from exposure to air pollutants in the short term may lead to headaches, dizziness, fatigue, and sinus irritation [3], while long term effects may lead to respiratory disease, heat disease, and cancer [3]. The pollutants may have a greater effect on people suffering from asthma, the elderly, and children. One of the easiest ways to reduce exposure to potentially harmful pollutants is through effective ventilation and providing fresh air to the occupied zone of buildings.

* Corresponding author: baskarg@iastate.edu 
Although there are many ways to ensure proper ventilation, a popular method that has been used in many applications is through measuring or calculation of the age of air [4]-[16]. The concepts of internal age of air, the air residual lifetime, and air residence time was first explored by Sandberg and Sjoberg [4] using tracer gas experiments. The internal age of air, $\tau_{i}$, is defined as the time required by a discrete fluid element to reach some point in the space since entering the room. The air residual life time, $\tau_{r l}$, is defined as the time required by a discrete fluid element to leave the room from an initial starting point in the room. The air residence time is the total time a fluid element spends in the room by passing through a given point in space. The concepts of age of air, residual life time, and residence time is pictorially represented in figure 1.

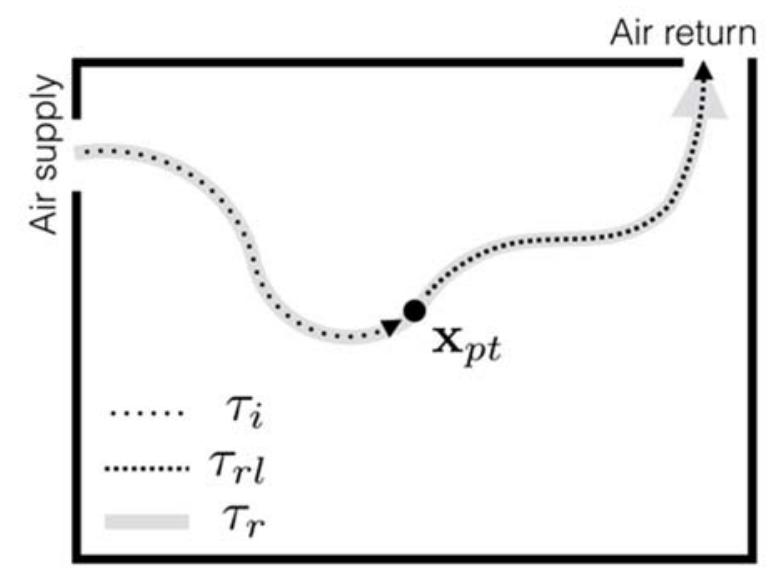

Figure 1: Description of the age of air, $\tau_{i}$, the air residual life time, $\tau_{r l}$, and air residence time, $\tau_{r}$ for a fluid element that passes through a given point, $\boldsymbol{x}_{\boldsymbol{p}}$, in the building.

Experimentally, age of air can be measured through tracer gas experiments [4], [5], [9], [13], [17] through a decay method, a source method, and a pulse method. These experimental techniques essentially determine the age of air through the response of the tracer gas concentration as the concentration is allowed to decay, applied as a constant source, and applied as a single pulse, respectively. Numerically, age of air has been calculated using an advection-diffusion partial differential equation (PDE) [6], [9]-[13], [15], [18]. This transport equation describes a fluid element being advected by an underlying flow field and that diffuses at a rate based on the laminar and turbulent Schmidt numbers along with laminar and turbulent viscosities. During advection, a source term (age) is added until the element leaves the space. Although the transport equation for age of air has been fairly well established to provide accurate estimates, the corresponding transport equation for air residual lifetime has not been well established. Both the age of air and the residual life time is needed to calculate the air residence time.

The computational approaches above are based on solving a PDE for the transport of contaminants in a room or building zone. Some recent work has been performed on a different method for contaminant transport, the Markov method [19]-[24]. The Markov method is a useful method for contaminant transport because 
it has been show to be faster than the PDE approach [23], [24]. The real-time prediction of contaminant propagation provides promise for demand response HVAC control. Applications of Markov matrices have also been shown in the areas of optimal sensor placement algorithms [25]-[27].

In this paper, the structure of Markov matrices is exploited to provide performance measures for use in mechanical ventilation system design. The purpose of this paper is to 1) establish two different approaches to calculate age of air, air residual life time, air residence time, and ventilation effectiveness, 2) show how both techniques are related, and 3) establish the mathematical relationship between residual life time and age of air. The numerical developments in this paper addresses the major gaps in analyzing mechanical system ventilation performance. These gaps in knowledge are 1) the need for a transport equation that describes the residual life time and subsequently the calculation of residence time and 2) the connection between continuous and discrete methods for calculating measures of mechanical system ventilation performance.

The two methods extended and developed in this paper are based on PDEs and Markov matrices. A new transport equation for residual life time is established that gives similar results to the Markov method for residual life time. The results of both the PDE method and the Markov method are compared with experimental data. The work presented in this paper provides a link between the PDE method and Markov methods and closes the loop for computationally calculating age of air, air residual life time, and air residence time to estimate aspects of mechanical ventilation system performance.

\section{Methods}

This section explains how a Markov matrix is used for contaminant transport, investigates the structure of the Markov matrices calculated for contaminant transport, and develops two different methods to quantify measures of mechanical ventilation system performance.

\subsection{Mathematical Preliminaries: Contaminant transport using Markov matrices}

A common well established computational method for tracking a particulate contaminant or a gas, $\phi$, in the indoor environment under some flow field, $\boldsymbol{U}$, is by solving the advection-diffusion partial differential equation (PDE) [28]-[34], eq. 1.

$$
\frac{\partial \phi}{\partial t}+\nabla \cdot(\boldsymbol{U} \phi)+\nabla^{2}(D \phi)=\boldsymbol{S}_{\phi}
$$

The advection-diffusion equation, shown to be a generalization of the infinite dimensional Perron-Frobenius operator [35], advects and diffuses a concentration of a contaminant on a continuous vector field from one time instant to another. In the discrete finite dimensional case, we consider a finite discretization of the room or 
building into a set of cells $\left\{\omega_{1}, \ldots, \omega_{n}\right\}$. These cells are also called states. For a specific cell $\omega_{k}$ the volumetric concentration of the contaminant is calculated, eq. 2 .

$$
\psi_{t}^{k}=\frac{1}{V_{\omega k}} \iiint_{\omega_{k}} \phi(x, y, z, t) d V, \quad k=1, \ldots, n
$$

The discrete finite dimensional Perron-Frobenius operator [25], [27] maps these volumetric average concentration of a set of cells across a time period, $\Delta t$. This map is known as a Markov matrix, eq. 3. A Markov matrix describes the probabilistic transition from an initial state to all other states during a timestep, $\Delta t$.

$$
\boldsymbol{P}=P_{i j}=\left[\begin{array}{cccc}
P_{11} & P_{12} & \cdots & P_{1 n} \\
P_{21} & P_{22} & \cdots & P_{2 n} \\
\vdots & \vdots & \vdots & \vdots \\
P_{n 1} & P_{n 2} & \cdots & P_{n n}
\end{array}\right]
$$

A Markov matrix is a probability matrix, which implies that the entries of the matrix are all non-negative, eq. 4.

$$
P_{i j} \geq 0 \text { for all } i, j
$$

Since a Markov matrix describes a probabilistic process, the row sum and/or the column sum of the matrix must be equal to 1.0. The rows of a right stochastic matrix sum to 1, eq. 5, and describes a probabilistic transition from an initial state $i$ to states in the domain.

$$
\sum_{j=1}^{n}\left(P_{i j}\right)=1 \quad \text { for all } \quad i
$$

The columns of a left stochastic matrix sum to 1 , eq. 6 , and describes a probabilistic transition from states in the domain into a given final state $j$.

$$
\sum_{i=1}^{n}\left(P_{i j}\right)=1 \quad \text { for all } j
$$

Using a right stochastic or a left stochastic matrix, the propagation of a contaminant concentration at time $t$ to a time $t+\Delta t$ is calculated by a simple matrix vector product, eq. 7.

$$
\boldsymbol{\psi}_{t+\Delta t}=\boldsymbol{\psi}_{t} \boldsymbol{P}
$$

For the calculation of Markov matrices for a given flow field two approaches have been developed: a flux based approach [21], [22] and a set theory based approach [24]. In this paper, the method for calculating the Markov matrix is not important, as long as the appropriate flow physics is contained in the Markov matrix. The physics of interest are accurate representations of advection and diffusion of air 
within a building, with the spatially varying diffusion rate based on the laminar and turbulent Schmidt numbers along with laminar and turbulent viscosities ${ }^{1}$, eq. 8.

$$
D=\left(\mu / \sigma_{l}+\mu_{t} / \sigma_{t}\right)
$$

\subsection{Canonical form of absorbing Markov matrices}

An absorbing Markov matrix is defined as having at least 1 absorbing state, and there is a non-zero probability that a non-absorbing (transient) state can reach an absorbing state in a finite number of steps. Figure 2 is an example of an absorbing Markov matrix. Absorbing states (like state 3, fig. 2) is a state that is impossible to leave. Absorbing states have a single entry of 1.0 on the diagonal, which implies that a contaminant in an absorbing state has a probability of 1.0 of staying in the current state in the next timestep. States that are not absorbing states are called transient states (like state 1 and state 2 , fig. 2 ). For ventilated systems the outlets serve as the absorbing states, and all other states are transient states. Due to diffusion each state is guaranteed to be connected to an outlet.

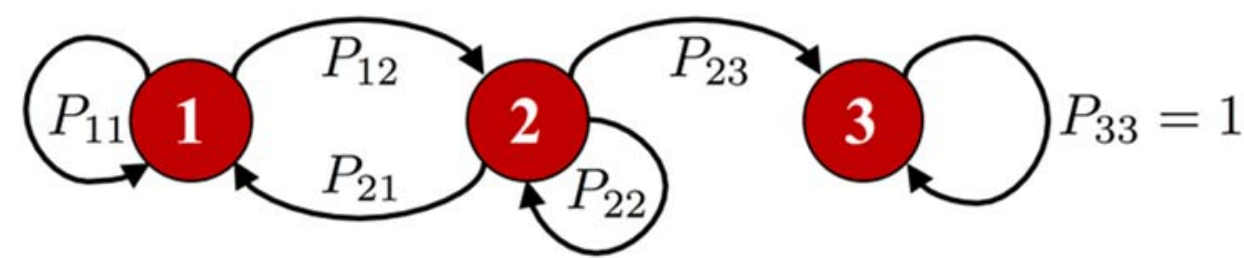

Figure 2: An example of an absorbing Markov matrix. State 3 is an absorbing state, while state 1 and 2 are transient states.

Absorbing Markov matrices have a standard 'canonical' form, eq. 9, where the transient states (TR) are grouped together and absorbing states (AB) are grouped together.

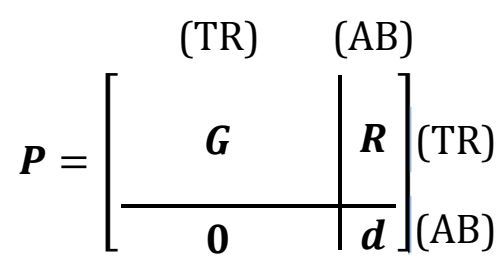

\footnotetext{
1 The particular method used to calculate the Markov matrices for this study involves 4 steps. 1) Initializing a given cell $\omega_{k}$ with a value of 1.0 and the rest of the domain to be 0.0 . 2) Solving equation 1 with a diffusivity equivalent to equation 8 for the desired time step of the Markov matrix. 3) Collecting the non-zero scalar concentrations into the $k^{\text {th }}$ row of a sparse Markov matrix, $\boldsymbol{P}$. 4) Performing this process for each $k=1: n$ to produce the transition probabilities of each row of the Markov Matrix.
} 
The matrix $\boldsymbol{G}$ is a $\left(n-n_{o}\right) \times\left(n-n_{o}\right)$ matrix that contains all the transient states, $\boldsymbol{R}$ is a $\left(n-n_{o}\right) \times n_{o}$ matrix that specifies which states will transition to an absorbing outlet (air return) state, the matrix $\boldsymbol{d}$ is a $n_{o} \times n_{o}$ identity matrix for the outlet states, and $\mathbf{0}$ is a $n_{o} \times\left(n-n_{o}\right)$ matrix containing all zeroes. The above decomposition of the finite dimensional Markov matrix into upper triangular matrix can also carry over to the infinite dimensional Perron-Frobenius (P-F) operator. We refer the reader to [36], [37] for the details on the decomposition of P-F operator.

\subsection{Quantify measures of IAQ}

In this section, two different methods for quantifying measures of mechanical system ventilation are developed. The first method is based on the more traditional transport equations (PDE method). The second method is using the Canonical form of absorbing Markov matrices (Markov method). Both methods are used to quantify internal age of air, air residual life time, air residence time, and ventilation efficiency.

\subsubsection{PDE method}

Computationally, age of air has been calculated using a steady state transport PDE [6], [9], [13], [18], [38], [39], eq. 10.

$$
\nabla \cdot\left[\rho \boldsymbol{U} \tau_{i}-\left(\mu / \sigma_{l}+\mu_{t} / \sigma_{t}\right) \nabla \tau_{i}\right]=1
$$

Physically, this equation represents a fluid element gaining a source concentration (age) while being advected along the velocity field, $\boldsymbol{U}$, and diffusing at a rate proportional to the effective viscosity at each point in the room. Some references use $\sigma_{l}=\sigma_{t}=0.7$ [11], [13], while recent work gives an alternate method for calculating the turbulent Schmidt numbers in stratified flows [40]. The boundary conditions of this equation are $\tau_{i}=0$ at all inlets and $\partial \tau_{i} / \partial \boldsymbol{x}=0$ at all the outlets and the walls.

Although this transport equation has been used extensively, a transport equation for air residual life time has not been established. Equation 11, is similar to the age of air equation, except the velocity field has been reversed. This transport equation represents the movement of a passive discrete fluid element from the outlet into the room along the reverse velocity field, $-\boldsymbol{U}$. The fluid element gains a source concentration (residual life) and diffuses at the rate of the effective viscosity at each point in the room as the fluid element moves further away from the outlet. The boundary conditions of this equation are $\tau_{r l}=0$ at all outlets and $\partial \tau_{r l} / \partial \boldsymbol{x}=0$ at all the inlets and the walls.

$$
\nabla \cdot\left[\rho(-\boldsymbol{U}) \tau_{r l}-\left(\mu / \sigma_{l}+\mu_{t} / \sigma_{t}\right) \nabla \tau_{r l}\right]=1
$$

Remark: Mathematically, this equation is the adjoint [41] of equation 10. Looking at Figure 1, it is clear that $\tau_{r l}$ can be calculated by starting at the outlet and moving backwards in time. The adjoint equation formally captures this intuition. 
Once the internal age of air and the residual life time fields have been calculated, other quantities of mechanical system ventilation performance can be calculated. The residence time relates both the age of air and the air residual life time, eq. 12.

$$
\tau_{r}=\tau_{i}+\tau_{r l}
$$

Another measure for mechanical ventilation system performance is ventilation effectiveness, eq. 13. Ventilation effectiveness [17] is calculated by the time constant of the room or building, $\tau$, and the age of air. The ventilation effectiveness provides a notion of the ventilation systems ability to provide fresh air into the space [17]. A value of 1.0 indicates that the system is well mixed, a value less than 1.0 indicates less than perfect mixing (stagnation or short-circuiting flow), and a value greater than 1 indicates the space is experiencing air flow similar to displacement ventilation or plug flow [17].

$$
\varepsilon_{I}=\tau / \tau_{i}
$$

\subsubsection{Markov method}

In the context of absorbing Markov matrices, the residual life time is related to the fundamental matrix. The fundamental matrix of an absorbing Markov matrix describes the average number of times state $i$ visits state $j$ before being absorbed [42], eq. 14.

$$
\boldsymbol{N}=N_{i j}=(\boldsymbol{I}-\boldsymbol{G})^{-1}
$$

The row sum of the fundamental matrix represents the total number of steps taken by a given state before it is absorbed. The row sum multiplied by the timestep (associated with the Markov matrix) provides the time duration until that state is absorbed (residual life time), eq. 15.

$$
\tau_{r l}=\Delta t \sum_{j=1}^{n-n_{o}} N_{i j}=\Delta t \boldsymbol{N} \mathbf{1}
$$

The calculation of $\tau_{r l}$ in equation 15 involves taking an inverse of a potentially large matrix $\boldsymbol{G}$ which computationally takes $O\left[\left(n-n_{o}\right)^{3}\right]$ operations [43]. Since this becomes computationally demanding for large matrices, a linear system of equations can be formulated by substituting equation 14 into equation 15.

$$
\begin{aligned}
& \tau_{r l}=\Delta t(\boldsymbol{I}-\boldsymbol{G})^{-1} \mathbf{1} \\
=>\quad & (\boldsymbol{I}-\boldsymbol{G}) \tau_{r l}=\Delta t \mathbf{1}
\end{aligned}
$$


The result is a linear system for the unknown $\tau_{r l}$ with coefficient matrix $(\boldsymbol{I}-\boldsymbol{G})$ and solution vector $\Delta t \mathbf{1}$. Solving the linear system is computationally more efficient (both memory and flops) and takes $\sim O\left[\left(n-n_{o}\right)^{2}\right]$ operations using standard linear solvers.

In the context of Markov matrices, reverse advection is performed by using the transpose of the Markov matrix, $\widetilde{\boldsymbol{P}}=\boldsymbol{P}^{T}$ (this is also equivalent to taking the adjoint [41] and in the infinite dimensional setting this adjoint operator is also known as Koopman operator. Spectral analysis of Koopman operator is used for dynamical system analysis and for solving problems involved in building systems applications [44]-[46]). The corresponding canonical form for the reverse advection Markov matrix is shown in equation $17 .^{2}$

$$
\widetilde{P}=\left[\begin{array}{c|c}
\widetilde{G} & \widetilde{\mathbf{0}} \\
\hline \widetilde{R} & \widetilde{d}
\end{array}\right]
$$

The matrix $\widetilde{\boldsymbol{G}}$ is a $\left(n-n_{i}\right) \times\left(n-n_{i}\right)$ matrix that contains all the transient states, $\widetilde{\boldsymbol{R}}$ is a $n_{i} \times\left(n-n_{i}\right)$ matrix that specifies which states will transition to the absorbing inlets states, the matrix $\widetilde{\boldsymbol{d}}$ is a $n_{i} \times n_{i}$ identity matrix for the inlet states, and $\widetilde{\mathbf{0}}$ is a $n-\left(n_{i} \times n_{i}\right)$ matrix containing all zeroes. Similar to the forward advection case, the same techniques in equations $14-16 \mathrm{~b}$ can be used on the reverse advection Markov matrix in equation 17 to produce a linear system of equation for the internal age or air, $\tau_{i}$, eq. 18 .

$$
(\boldsymbol{I}-\widetilde{\boldsymbol{G}}) \tau_{i}=\Delta t \mathbf{1}
$$

Once the residual life time field and internal age of air field are calculated by equation $16 \mathrm{~b}$ and equation 18 respectively, residence time and ventilation effectiveness can be calculated using the equations 12 and 13 respectively. $\tau_{i}$ has an infinite dimensional analog obtained using the infinite dimensional Perron-Frobenius operator. In particular, this infinite dimensional analog can be obtained by solving the linear advection PDE, i.e., eq. (1), or discrete time approximation of the PDE. In [36], this infinite dimensional analog is rigorously defined and is used as a stability certificate for verifying weaker notion of almost everywhere stability of the absorbing state.

\section{Flow field and validation}

\footnotetext{
${ }^{2}$ The rows containing the transitions for the inlets and the outlets may need to be
} swapped after the transpose. 
The room considered in this study is a $4.2(\mathrm{~m}) \times 3.0(\mathrm{~m})$ × $3.6(\mathrm{~m})$ room, fig. 3 , that has been used in previous age of air studies [9], [13]. Both the inlet and outlet dimensions are $0.2(\mathrm{~m}) \times 0.3(\mathrm{~m})$. The longer side of the inlet and outlet is along the $\mathrm{z}$-direction, figure 3 . The bottom of the inlet and outlet are positioned symmetrically along the center of the z-axis. The bottom edge of the inlet is $2.05(\mathrm{~m})$ above the floor. The ventilated room has an air change rate of $8(\mathrm{ACH})$, which corresponds to the room time constant $\tau=0.125(\mathrm{hr})$ and a constant inlet air speed of $1.68(\mathrm{~m} / \mathrm{s})$. The air flow in this space is considered to be isothermal [9].

The air flow in the ventilated room was computed by solving the Navier-Stokes equations. The Reynolds number for this based on the inlet height of $0.2(\mathrm{~m})$ is approximately 21,800 and is considered to be turbulent. The Reynolds stresses were modeled using the RNG k- $\varepsilon$ model. The turbulent conditions at the inlet are based on a turbulent intensity of $14 \%$. The turbulent kinetic energy at the inlet was calculated by $k_{\text {inlet }}=\frac{3}{2}\left[\left|U_{i}\right| I_{t}\right]^{2}$. The kinetic energy dissipation rate at the inlet was calculated by $\varepsilon_{\text {inlet }}=\left(k_{\text {inlet }}\right)^{1.5} /\left(0.005 \sqrt{A_{\text {inlet }}}\right) \cdot^{3}$

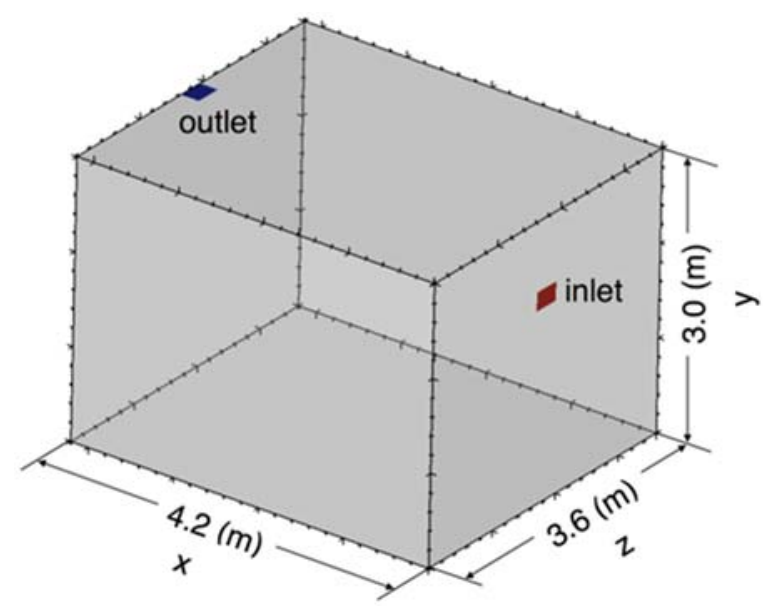

Figure 3: The geometry for the building used in this study.

The geometry, fig. 3, was discretized into 2 different meshes to investigate the effects of spatial convergence. Due to symmetry of the problem, only half of the domain was discretized. The first (coarse) discretization contains 22,680 hexahedra elements with sides approximately $0.1(\mathrm{~m})$, fig. $4 \mathrm{a}$. The second (fine) discretization contains 181,440 hexahedra elements with sides approximately 0.05 (m), fig. $4 \mathrm{~b}$.

3 For more information about the room setup, numerical simulations, and the experiments the readers are referred to work of M. Bartak et al., 2001 [9]. 

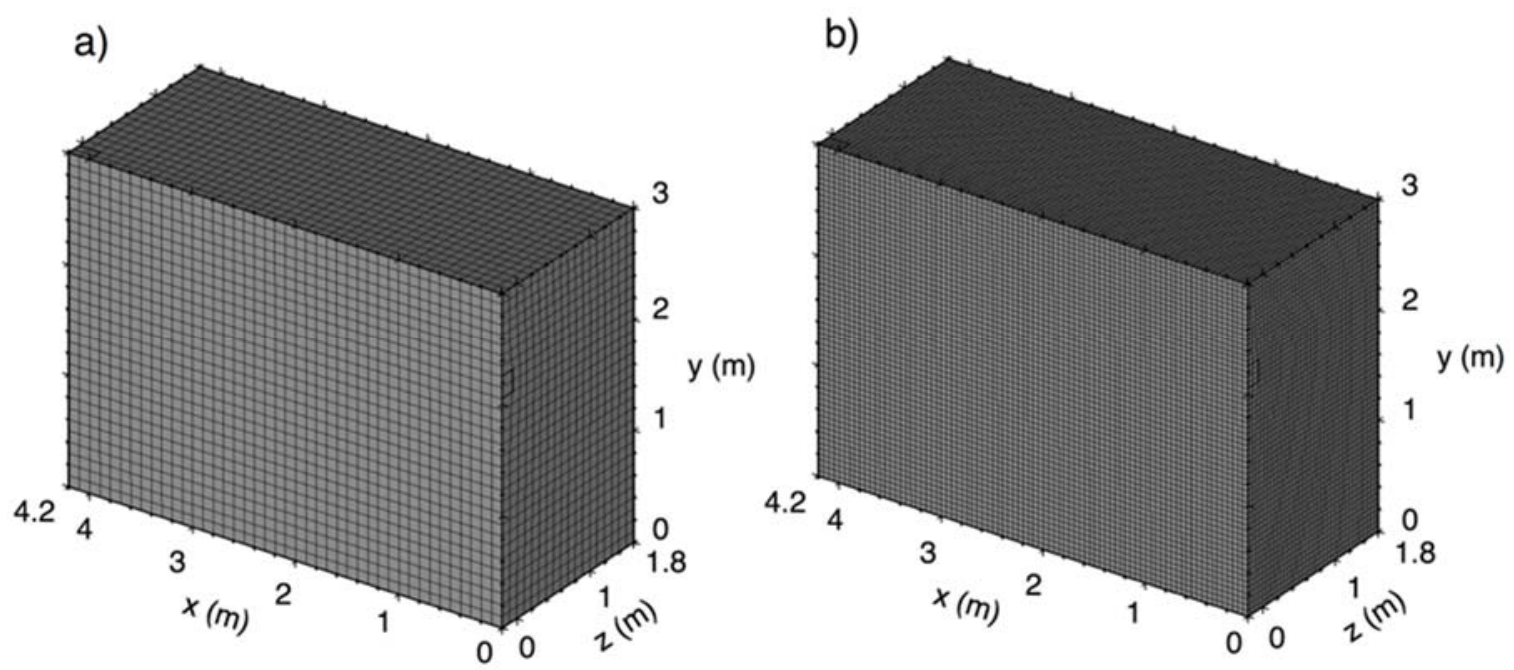

Figure 4: The a) coarse (22,680 elements) and b) fine (181,440 elements) discretization of the ventilated room.

The Navier-Stokes equations, turbulent transport equations, age of air transport equation (eq. 10), and the residual life time transport equation (eq. 11) were solved on both discretization to a residual tolerance of 1e-6. After the simulations converged, data was sampled along the symmetry plane $(\mathrm{z}=0)$ at three different $x$ locations $[x=1.13(\mathrm{~m}), \mathrm{x}=2.20(\mathrm{~m})$, and $\mathrm{x}=3.2(\mathrm{~m})]$ from the floor to the ceiling. The normalized age of air data from the simulations along with the experimental and numerical data from Bartak et al. [9] can be seen in figure 5. The normalizing constant is 538 seconds 4 . From these results, figure 5, the age of air calculated in this study is fairly accurate compared to the experimental data, and in generally performs better than the previous study. This reason may be due to the increased discretization used in the current study as there is a decrease in the age of air as the discretization is increased, figure 5 . Since the coarse mesh representation performs reasonably well compared to the fine mesh discretization, the coarser representation will be used to display the results for the rest of the paper.

\section{Results}

This section provides comparisons between experimental data and numerical results for both the PDE method and Markov method for the quantities of interest. The air residual life time and age of air are compared first as they are quantities directly solved by the transport PDEs (eq. 10 and 11) or a linear system from the Markov matrix (eq. 16b and 18). Since air residence time and ventilation efficiency are post-processed values, these quantities are compared after the age of air and residual life time comparison.

\footnotetext{
4 This normalizing constant of 538 seconds is used for all the normalized data presented in this paper.
} 

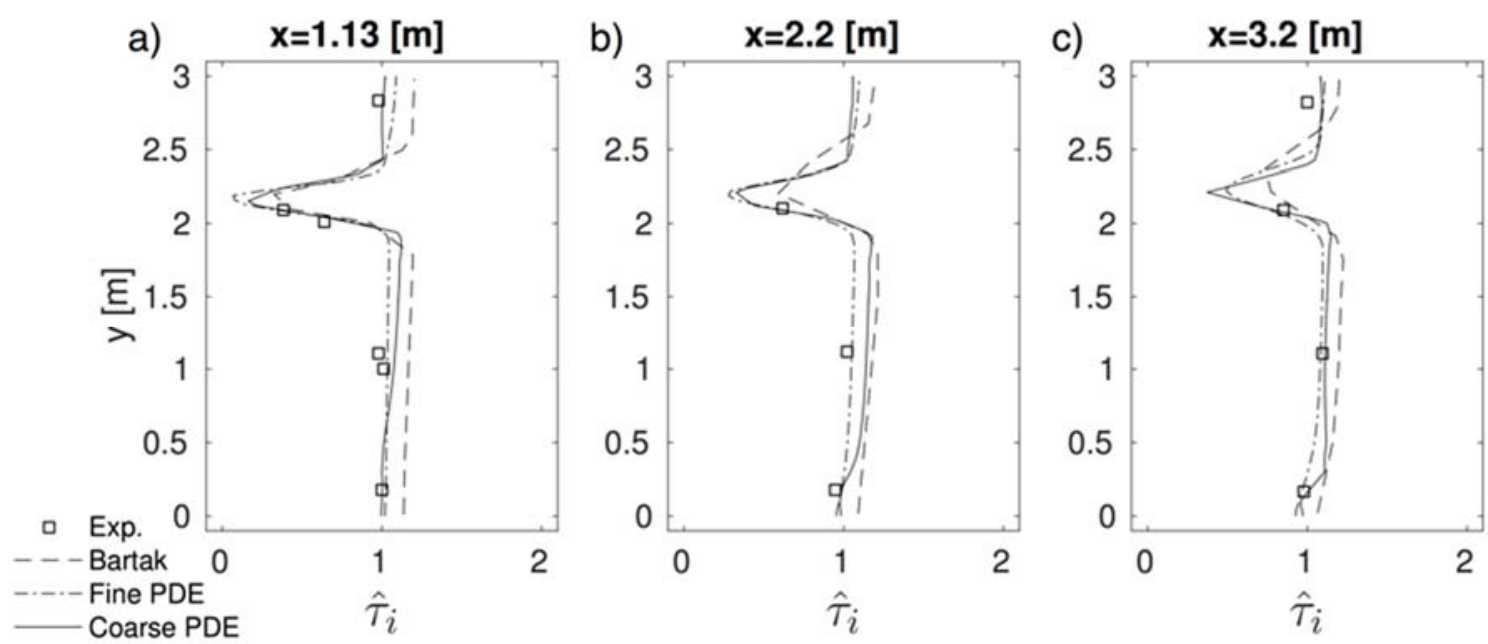

Figure 5: Normalized experimental and numerical age of air based on the equation 9 along three $x$-locations, a) $x=1.13(\mathrm{~m}), b) x=2.2(\mathrm{~m})$, and c) $x=3.2(\mathrm{~m})$.

\subsection{Air residual life time and age of air comparison}

The age of air and air residual life time are calculated by both the PDE method and the method using Markov matrices. Contour plots show the normalized isocontours of the age of air and residual life time in figure 6. The age of air, figure 6a and $6 \mathrm{c}$, is small near the inlet and increases as the jet carries the air all the way to the opposite all. Once the air hits the opposite wall, the air spreads out into the rest of the domain. For the regions away from the jet, the age is roughly constant. The normalized mean age of air in the room for both the PDE and Markov methods is calculated to be 0.97 and 1.00 respectively. The residual life time of the air is small near the ceiling, figure $6 \mathrm{~b}$ and $6 \mathrm{~d}$, which suggests that the air needs to reach the top center part of the room before it has a chance to leave. The areas near the far wall $[z \approx 1.8(m)]$ has a higher residual life time as the air slowly circulates back to the center of the space. The normalized mean residual life time in the room for both the PDE and Markov methods is calculated to be 1.00 for both methods. Sensitivity of the results to a change in the timestep associated with the Markov matrix can be see in appendix A.

The results of the Markov method and the PDE method for simulating internal age of air and air residual life time is compared in figure 7. The results show that both the PDE method and the Markov method provide fairly good agreement with the experimental age of air data in figure $7 \mathrm{a}-7 \mathrm{c}$. At these locations in the domain the Markov method performs slightly better than the PDE method. The largest differences in age of air for the two methods is near the ceiling. For air residual life time both the PDE approach and the Markov method essentially give the same result, figure 7d-7f. Based on these results, both the PDE method and the Markov method seem to provide reasonably good estimates for age of air and roughly equivalent measures of calculating air residual life time. 

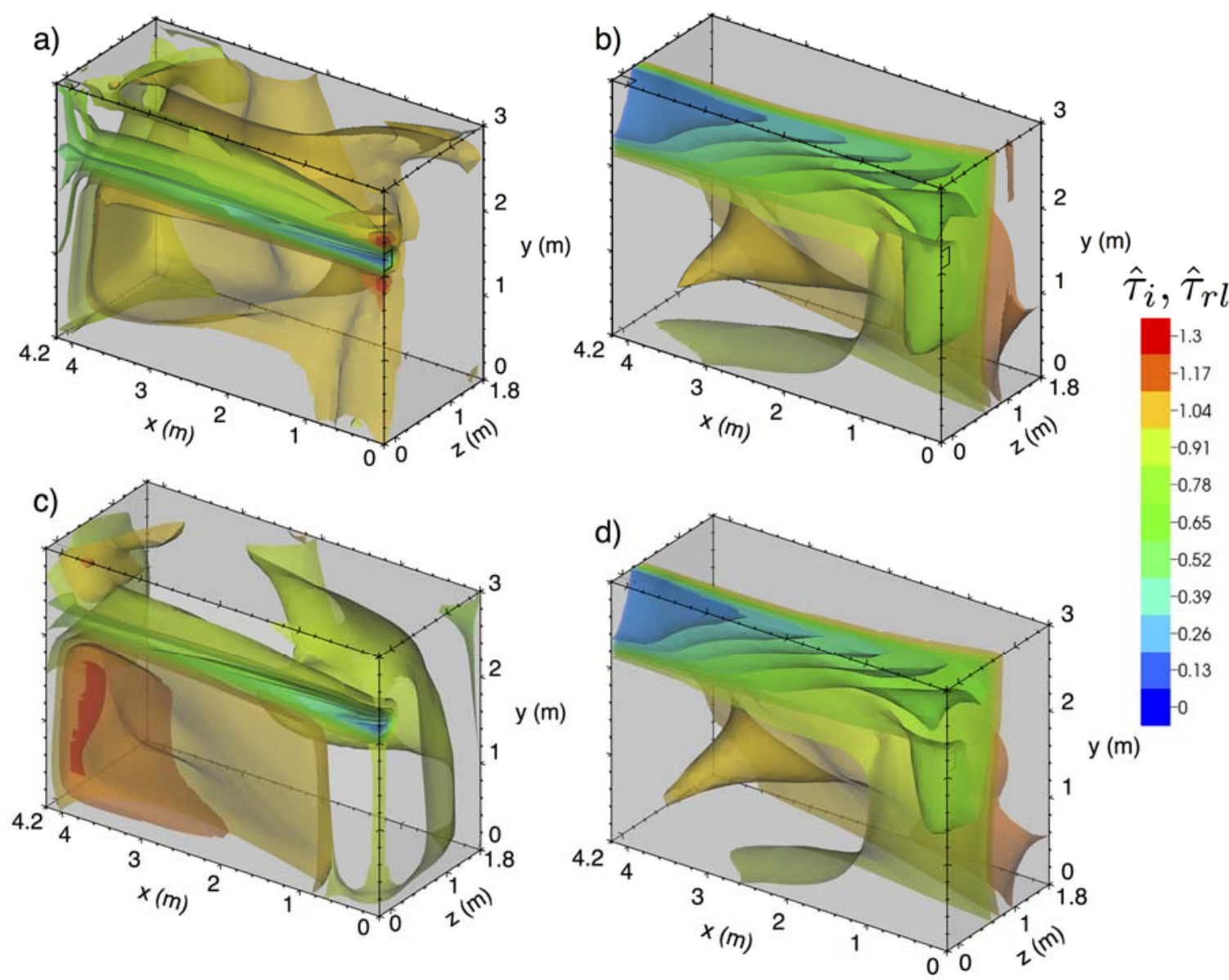

Figure 6: Normalized iso-contours of the a) the internal age of air field using the Markov method, b) the residual life time field using the Markov method, c) the internal age of air field using the PDE method, d) the residual life time field using the PDE method.

\subsection{Air residence time and ventilation effectiveness}

The previous subsection showed that the Markov method and the PDE methods provide similar estimates of internal age of air and air residual life time. This section compares the performance of the Markov method and the PDE method for measures of IAQ based on the age of air and air residual life time. The two quantities of interest are the residence time and ventilation effectiveness, figure 8 . The regions of the space with highest residence times are near the far wall $[z \approx 1.8(\mathrm{~m})]$, figure $8 \mathrm{a}$ and $8 \mathrm{c}$. This is not surprising as this region has the largest residual life times and age of air. The normalized mean residence time in the room for both the PDE and Markov methods is calculated to be 1.97 and 2.00 respectively. For ventilation effectiveness the region near the jet has very high ventilation effectiveness, due to the high air speed of this region, figure $8 \mathrm{~b}$ and $8 \mathrm{~b}$. The rest of the domain is a ventilation effectiveness of about 0.8 . The mean ventilation effectiveness in the room for both the PDE and Markov methods is calculated to be 1.02 and 0.90 respectively. 

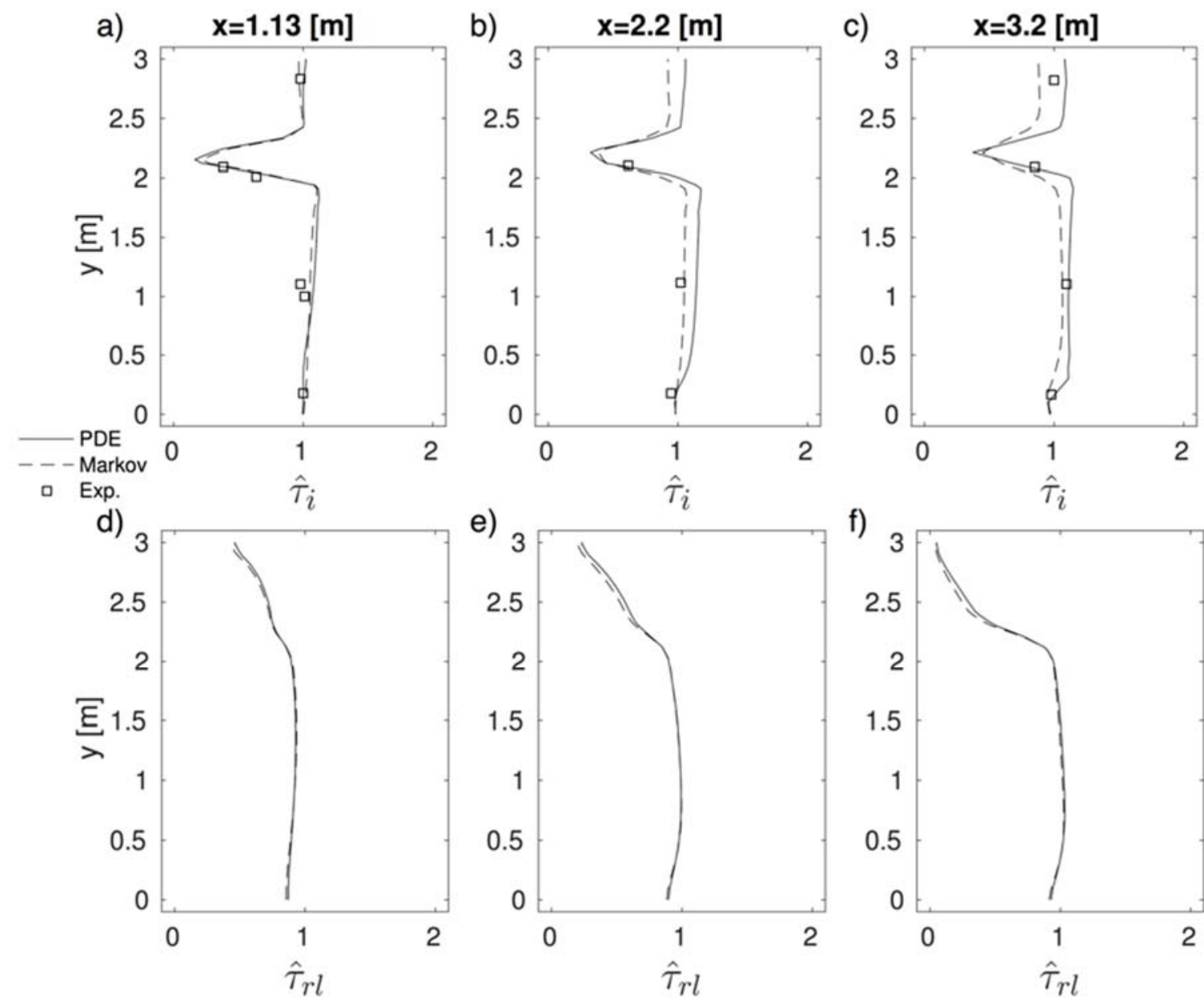

Figure 7: Normalized internal age of air comparisons at different $x$-locations along the $z$ symmetry plane for $a$ ), $b$ ), and $c$ ). Normalized comparisons of air residual life time at different $x$-locations along the $z$ symmetry plane for $d$ ), e), and f).

Residence time and ventilation efficiency calculated using the Markov method and the PDE method are compared in figure 9. The two methods essentially provide the same results for residence time with the exception of regions near the ceiling, figure $9 \mathrm{a}-9 \mathrm{c}$. The difference between the two techniques is due to the differences seen in the internal age of air in figure 7. For ventilation effectiveness, the two methods roughly provide the same estimates of ventilation effectiveness, figure $9 \mathrm{~d}-$ 9f. The Markov method shows a slight decrease in the peak for each location at the center of the jet. Once again, a slight difference in the ventilation effectiveness is shown near the ceiling, where the Markov method predicts a slightly higher ventilation effectiveness. Overall, the two methods provide similar predictions for both the residence time and the ventilation effectiveness. 

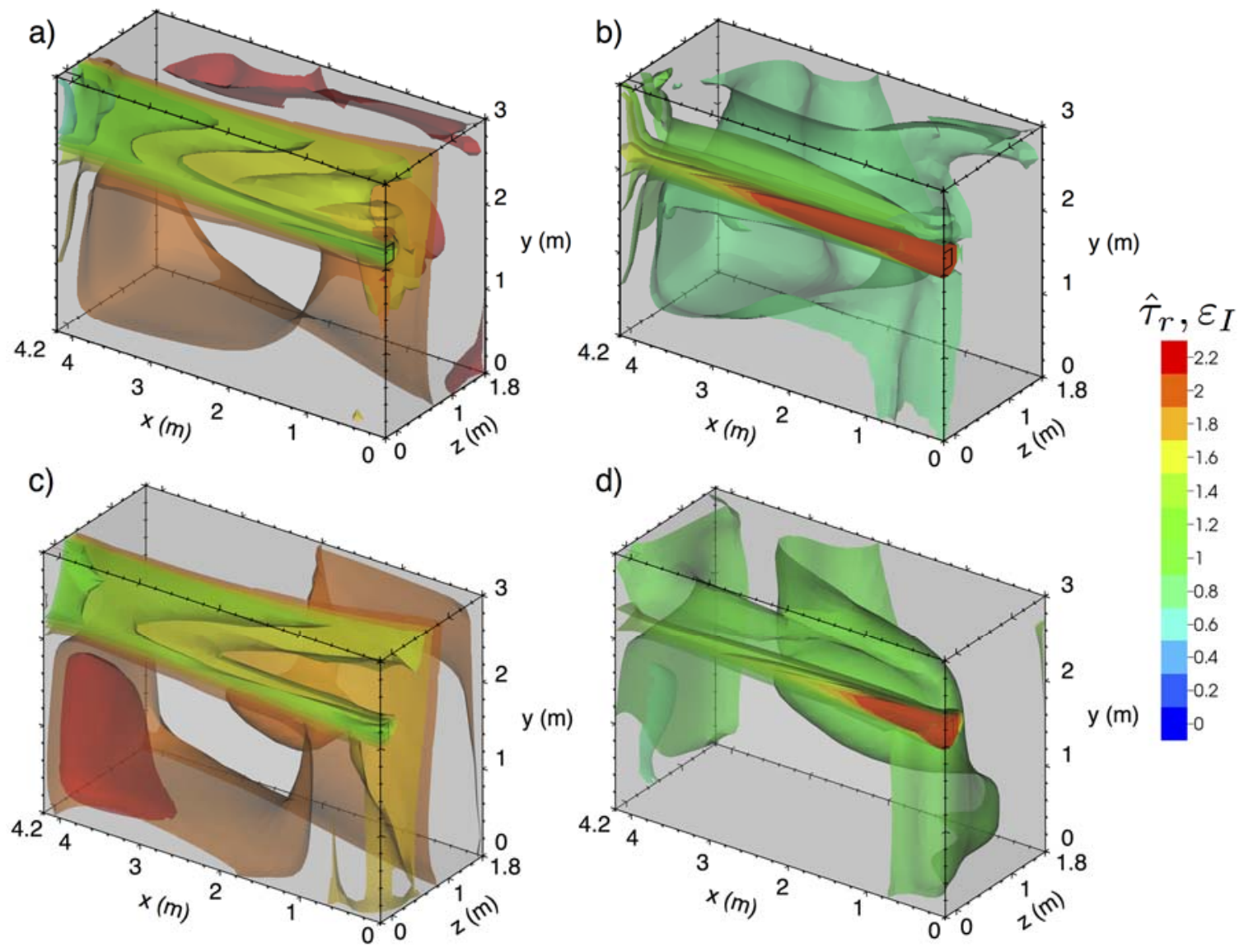

Figure 8: Normalized iso-contours of the a) the residence time field using the Markov method, $b$ ) the ventilation effectiveness using the Markov method, c) the residence time field using the PDE method, d) the ventilation effectiveness using the PDE method. 

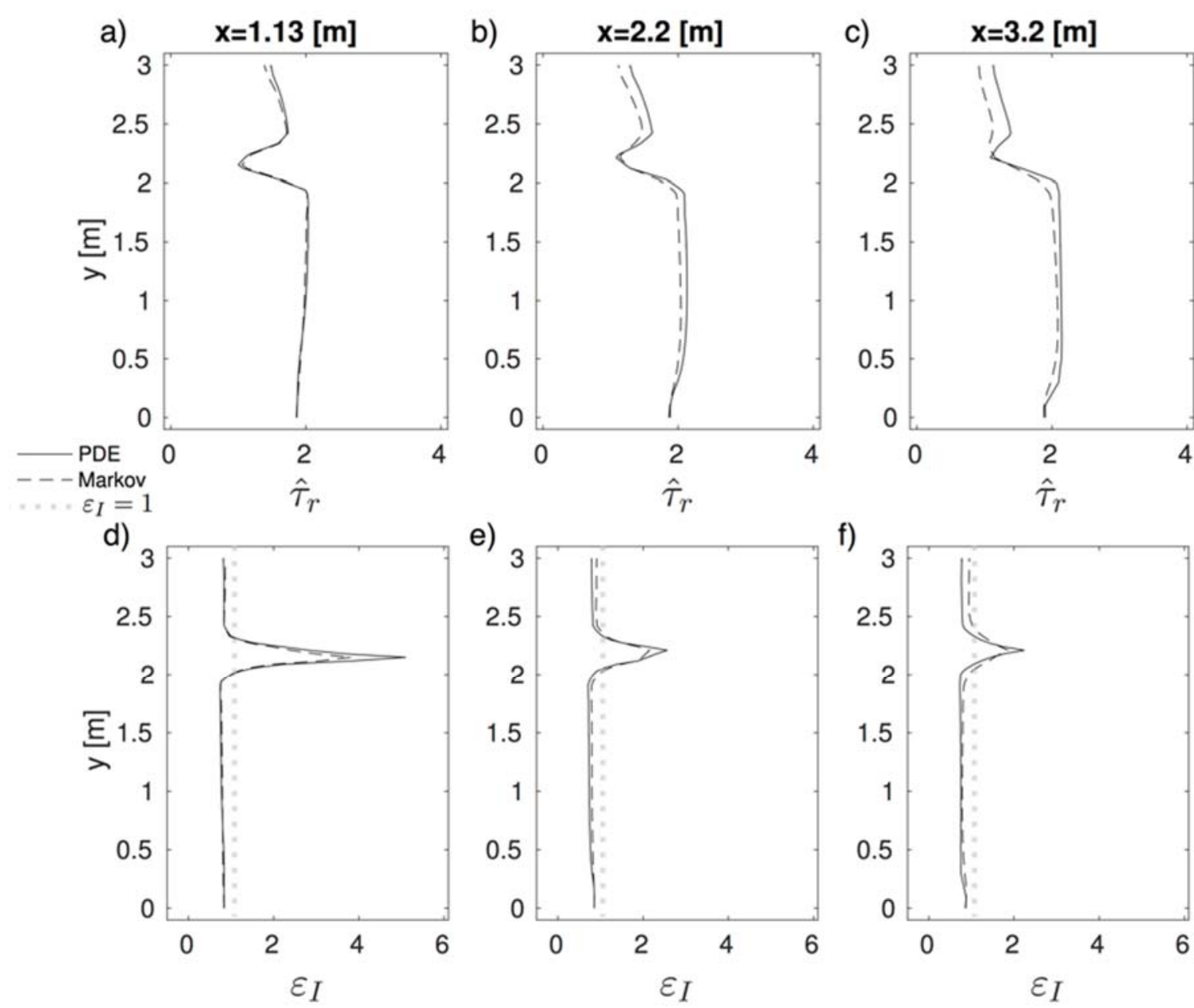

Figure 9Normalized residence time comparisons at different $x$-locations along the $z$ symmetry plane for $a$ ), $b$ ), and $c$ ). Ventilation effectiveness at different $x$-locations along the z symmetry plane for $d$ ), e), and f).

\section{Discussion}

This study used a validated benchmark problem to explore the connection between continuous and discrete methods for calculating performance measures of mechanical system ventilation. We show that these two alternate approaches yield equivalent and accurate results. A new transport equation for residual life time is developed. The new equation is the adjoint of the age of air transport equation. This mathematical connection (in both the continuous and discrete setting) opens up the utility of a variety of mathematics tools in linear control theory that can be applied to the analysis of mechanical ventilation systems. The introduction of an equation for residual lifetime also allows for residence time to be calculated.

Just as the transport equations provide a continuous representation of these metrics, Markov methods are shown to provide the corresponding discrete representation of these metrics. We identified the fact that previous studies [9], [13] 
calculating the age of air over predicted the values due to coarse mesh resolutions. These advances show the importance of spatial convergence and provide a complete picture of the relationships between continuous and discrete methods for calculating mechanical system performance.

The two different approaches developed in this paper have their own advantages and disadvantages. The advantage of using Markov based methods is their broad applicability beyond just mechanical system performance. If a Markov based method is utilized to quantify mechanical system performance, then the resultant data can be subsequently used to determine optimal sensor locations [47], perform fast contaminant transport [22], [23], [48] and build estimators for demand control, and for rapid contaminant source detection. A potential disadvantage, however, is the increased computational effort needed in calculating the Markov matrices. In contrast, for the PDE method, the passive scalar transport equations for age of air and residual life time calculations can be easily solved for when the flow field is being solved with very little additional computational overhead [49], [50]. The disadvantage, in turn, is the lack of broader applicability beyond mechanical ventilation system performance assessment (like contaminant transport, sensor placement, and source identification) which Markov based methods can naturally perform.

\section{Conclusions}

This paper expands and develops two different methods for quantifying mechanical ventilation system performance. The first method uses transports equations and the second method uses the structure of absorbing Markov matrices to calculate age of air and air residual life time. The methods are validated with experimental tracer gas data for a ventilated room. We have shown that the Markov method is the discrete counterpart of the PDE based method, that the residual life time and age of air are related through adjoint equations, and have established a transport equation (and its discrete counterpart) for residual life time to quantify mechanical ventilation system performance. Both Markov (discrete) and PDE (continuous) methods show similar results for age of air and residual life time along with the processed quantities of residence time and ventilation effectiveness. Based on these results both methods are valid and useful for calculating age of air, air residual life time, air residence time, and ventilation efficiency. We advocate the use of the PDE method when only system performance assessment is needed, as it has minimal computational overhead over the CFD simulation required to compute the flow field. If a more integrated approach to building analysis is taken -- including contaminant release scenario assessment, emergency response planning, or sensor placement strategies - then we advocate the use of the Markov method approach. This work also provides approaches for integrating residual life time and residence time into the mechanical system design process. Using these methods designers, architects, and researchers can obtain a better understanding of the ventilation 
performance of rooms and buildings they design, thus ensuring safety and comfort while potentially minimizing energy usage.

\section{Acknowledgements}

BG and AF acknowledge NSF 1149365 and NSF 1306866 for partial support. Computing support from NSF via XSEDE resource grant CTS110007 is gratefully acknowledged.

\section{Nomenclature}

\begin{tabular}{|c|c|c|c|}
\hline \multirow{2}{*}{$\begin{array}{l}A_{\text {inlet }} \\
D\end{array}$} & \multicolumn{2}{|l|}{ Area of the inlet } & \\
\hline & $\begin{array}{l}\text { Diffusivity of the contaminant in } \\
\text { air } \\
\text { An identity matrix of size } n-\end{array}$ & $\boldsymbol{S}_{\phi}$ & $\begin{array}{l}\text { reverse transport Markov matrix } \\
\text { Spatially dependent contaminant } \\
\text { source term }\end{array}$ \\
\hline & $n_{o} \times n-n_{o}$ & $t$ & Time \\
\hline$\widetilde{\boldsymbol{d}}$ & An identity matrix of size $n-$ & $\boldsymbol{U}$ & Air flow velocity field \\
\hline $\boldsymbol{G}$ & $\begin{array}{l}n_{i} \times n-n_{i} \\
\text { The matrix of the transition }\end{array}$ & $\left|U_{i}\right|$ & $\begin{array}{l}\text { The air velocity magnitude at the } \\
\text { inlet }\end{array}$ \\
\hline & probabilities between transient & $V_{\omega_{k}}$ & Volume of state $\omega_{k}$ \\
\hline & states of the forward transport & $x$ & The $\mathrm{x}$ direction \\
\hline & Markov matrix & $x$ & The position vector $(x, y, z)$ \\
\hline$\widetilde{\boldsymbol{G}}$ & The matrix of the transition & $y$ & The y direction \\
\hline & probabilities between transient & $Z$ & The $\mathrm{z}$ direction \\
\hline & $\begin{array}{l}\text { states of the reverse transport } \\
\text { Markov matrix }\end{array}$ & $\Delta t$ & $\begin{array}{l}\text { The timestep associated with the } \\
\text { Markov matrix }\end{array}$ \\
\hline $\boldsymbol{I}$ & An identity matrix & $\varepsilon_{\text {inlet }}$ & Turbulent kinetic energy \\
\hline$I_{t}$ & The turbulent intensity at the & & dissipation at the inlet \\
\hline & inlet & $\varepsilon_{I}$ & Ventilation effectiveness \\
\hline$k_{\text {inlet }}$ & $\begin{array}{l}\text { Turbulent kinetic energy at the } \\
\text { inlet }\end{array}$ & & Dynamic viscosity \\
\hline$N$ & $\begin{array}{l}\text { The fundamental matrix of the } \\
\text { forward transport Markov matrix }\end{array}$ & $\begin{array}{l}\mu_{t} \\
\rho \\
\sigma_{l}\end{array}$ & $\begin{array}{l}\text { Dynamic turbulent viscosity } \\
\text { The density of air } \\
\text { The laminar Schmidt number }\end{array}$ \\
\hline$n$ & The number of Markov states & $\sigma_{t}$ & The turbulent Schmidt number \\
\hline$n_{o}$ & $\begin{array}{l}\text { The number of outlet Markov } \\
\text { states }\end{array}$ & $\begin{array}{l}\tau \\
\tau_{i}\end{array}$ & $\begin{array}{l}\text { The room time constant } \\
\text { The internal age of air }\end{array}$ \\
\hline $\boldsymbol{P}$ & $\begin{array}{l}\text { The forward transport Markov } \\
\text { matrix }\end{array}$ & $\hat{\tau}_{i}$ & $\begin{array}{l}\text { The internal age of air normalized } \\
\text { by } 538(\mathrm{sec})\end{array}$ \\
\hline$\widetilde{\boldsymbol{P}}$ & $\begin{array}{l}\text { The reverse transport Markov } \\
\text { matrix }\end{array}$ & $\tau_{r}$ & The air residence time \\
\hline $\boldsymbol{R}$ & $\begin{array}{l}\text { matrix } \\
\text { The matrix of the transition }\end{array}$ & $\hat{\tau}_{r}$ & $\begin{array}{l}\text { The air residence time normalized } \\
\text { by } 538(\mathrm{sec})\end{array}$ \\
\hline & probabilities between transient & $\tau_{r l}$ & The air residual life time \\
\hline & $\begin{array}{l}\text { states and absorbing states for the } \\
\text { forward transport Markov matrix }\end{array}$ & $\hat{\tau}_{r l}$ & $\begin{array}{l}\text { The air residual life time } \\
\text { normalized by } 538(\mathrm{sec})\end{array}$ \\
\hline$\widetilde{\boldsymbol{R}}$ & $\begin{array}{l}\text { The matrix of the transition } \\
\text { probabilities between transient }\end{array}$ & $\phi$ & $\begin{array}{l}\text { Continuous contaminant } \\
\text { concentration }\end{array}$ \\
\hline
\end{tabular}


$\psi_{t}^{k} \quad$ Discrete volumetric average concentration in state $k$ at time $t$

$\omega_{k} \quad$ The $k^{\text {th }}$ Markov state

0 A matrix of size $n_{o} \times n-n_{o}$ containing all zeroes $\widetilde{0} \quad$ A matrix of size $n_{i} \times n-n_{i}$ containing all zeroes

1 A vector with all elements equal to 1.0

|. $\quad$ The magnitude operator

\section{References}

[1] U.S. EPA/Office of Air and Radiation, "The Inside Story: A Guide to Indoor Air Quality," Office of Radiation and Indoor Air (6609J) Cosponsored with the Consumer Product Safety Commission, EPA 402-K93-007., 2012. [Online]. Available: http://www.epa.gov/iaq/pubs/insidestory.html. [Accessed: 08-Jul2015].

[2] World Health Organization, "Combined or multiple exposure to health stressors in indoor built environments," 2013.

[3] U.S. EPA, “EPA Strategic Plan," Washington, D.C., 2014.

[4] M. Sandberg and M. Sjöberg, "The use of moments for assessing air quality in ventilated rooms," Build. Environ., vol. 18, no. 4, pp. 181-197, 1983.

[5] M. H. Sherman, "Tracer-gas techniques for measuring ventilation in a single zone," Build. Environ., vol. 25, no. 4, pp. 365-374, 1990.

[6] G. Gan and H. B. Awbi, "Numerical prediction of the age of air in ventilated rooms," ROOMVENT, vol. 94, no. 16-27, 1994.

[7] C. C. Federspiel, "Air-Change Effectiveness : Theory and Calculation," Indoor Air, vol. 9, pp. 47-56, 1999.

[8] H. Xing, A. Hatton, and H. B. Awbi, "A study of the air quality in the breathing zone in a room with displacement ventilation," Build. Environ., vol. 36, pp. 809-820, 2001.

[9] M. Bartak, M. Cermak, J. a Clarke, J. Denev, F. Drkal, M. Lain, I. a Macdonald, M. Majer, and P. Stankov, "Experimental and Numerical Study of Local Mean Age of Air," 7th Int. Build. Perform. Simul. Assoc. Conf., pp. 773-780, 2001.

[10] X. Li, D. Li, X. Yang, and J. Yang, "Total air age: An extension of the air age concept," Build. Environ., vol. 38, no. 11, pp. 1263-1269, 2003.

[11] J. Abanto, D. Barrero, M. Reggio, and B. Ozell, "Airflow modelling in a computer room," Build. Environ., vol. 39, no. 12, pp. 1393-1402, 2004.

[12] Z. Lin, T. T. Chow, K. F. Fong, C. F. Tsang, and Q. Wang, "Comparison of performances of displacement and mixing ventilations. Part II: indoor air quality," Int. J. Refrig., vol. 28, no. 2, pp. 288-305, 2005.

[13] V. Chanteloup and P. S. Mirade, "Computational fluid dynamics (CFD) modelling of local mean age of air distribution in forced-ventilation food 
plants," J. Food Eng., vol. 90, no. 1, pp. 90-103, 2009.

[14] C. Buratti, R. Mariani, and E. Moretti, "Mean age of air in a naturally ventilated office: Experimental data and simulations," Energy Build., vol. 43, no. 8, pp. 2021-2027, 2011.

[15] S. Gilani, H. Montazeri, and B. Blocken, "CFD simulation of stratified indoor environment in displacement ventilation: Validation and sensitivity analysis," Build. Environ., vol. 95, pp. 299-313, 2015.

[16] T. Zerihun Desta, S. Van Buggenhout, A. Van Brecht, J. Meyers, J.-M. Aerts, M. Baelmans, and D. Berckmans, "Modelling mass transfer phenomena and quantification of ventilation performance in a full scale installation," Build. Environ., vol. 40, no. 12, pp. 1583-1590, 2005.

[17] ASHRAE, ASHRAE Fundamentals: Ventilation and Infiltration. Atlanta, GA: American Society of Heating, Refigeration and Air-Conditioning Engineers, Inc., 2009.

[18] M. Bartak, I. Beausoleil-morrison, J. A. Clarke, J. Denev, F. Drkal, and M. Lain, "Integrating CFD and building simulation," vol. 37, pp. 865-871, 2002.

[19] R. M. Jones and M. Nicas, "Benchmarking of a Markov Multizone Model of Contaminant Transport," Ann. Occup. Hyg., vol. 58, pp. 1018-1031, 2014.

[20] R. M. Jones and M. Nicas, "Experimental Evaluation of a Markov Multizone Model of Particulate Contaminant Transport," Ann. Occup. Hyg., vol. 58, no. 8, pp. 1018-1031, 2014.

[21] C. Chen, W. Liu, C. Lin, and Q. Chen, "A Markov Chain Model for Predicting Transient Particle Transport in Enclosed Environments," Build. Environ. Accept. Manuscr., 2015.

[22] C. Chen, C. H. Lin, Z. Long, and Q. Chen, "Predicting transient particle transport in enclosed environments with the combined computational fluid dynamics and Markov chain method," Indoor Air, vol. 24, pp. 81-92, 2014.

[23] C. Chen, W. Liu, C. Lin, and Q. Chen, "Comparing the Markov Chain Model with the Eulerian and Lagrangian Models for Indoor Transient Particle Transport Simulations," Aerosol Sci. Technol., vol. 49, pp. 857-871, 2015.

[24] A. D. Fontanini, U. Vaidya, and B. Ganapathysubramanian, "Constructing Markov matrices for real-time transient contaminant transport analysis for indoor environments," Build. Environ., vol. 94, pp. 68-81, Dec. 2015.

[25] A. D. Fontanini, U. Vaidya, and B. Ganapathysubramanian, "A methodology for optimal placement of sensors in enclosed environments: A dynamical systems approach," Under Rev., 2015.

[26] S. Sinha, U. Vaidya, and R. Rajaram, "Actuator and sensor placement for control of non-equilibrium dynamics," Proc. Eur. Control Con-ference, pp. 1083-1088, 2013. 
[27] U. Vaidya, R. Rajaram, and S. Dasgupta, "Actuator and sensor placement in linear advection PDE with building system application," J. Math. Anal. Appl., vol. 394, no. 1, pp. 213-224, 2012.

[28] M. Bady, S. Kato, and H. Huang, "Towards the application of indoor ventilation efficiency indices to evaluate the air quality of urban areas," Build. Environ., vol. 43, pp. 1991-2004, 2008.

[29] C. a Gilkeson, C. J. Noakes, P. a Sleigh, M. a I. Khan, and M. a Camargo-Valero, "Simulating Pathogen Transport within a Naturally Ventilated Hospital Ward," World Acad. Sci. Eng. Technol., vol. 79, no. 7, pp. 119-125, 2011.

[30] P. Kassomenos, a. Karayannis, I. Panagopoulos, S. Karakitsios, and M. Petrakis, "Modelling the dispersion of a toxic substance at a workplace," Environ. Model. Softw., vol. 23, pp. 82-89, 2008.

[31] S. Kato and J. H. Yang, "Study on inhaled air quality in a personal airconditioning environment using new scales of ventilation efficiency," Build. Environ., vol. 43, pp. 494-507, 2008.

[32] J. Li, I. Yavuz, I. Celik, and S. Guffey, "Predicting Worker Exposure-The Effect of Ventilation Velocity, Free-Stream Turbulence and Thermal Condition," J. Occup. Environ. Hyg., vol. 4, no. 11, pp. 864-874, 2011.

[33] J. Srebric, J. Yuan, and A. Novoselac, "In-Situ Experimental Validation of a Coupled Multi-zone and CFD Model for Building Contaminant Transport Simulations," ASHRAE Trans., vol. 114(1), pp. 273-281, 2008.

[34] L. Wang and Q. Chen, "Validation of a Coupled Multizone-CFD Program for Building Airflow and Contaminant Transport Simulations," HVAC\&R Res., vol. 13, no. 2, pp. 267-281, 2007.

[35] L. Cortelezzi and I. Mezic, Analysis and Control of Mixing with an Application to Micro and Macro Flow. Springer Science \& Business Media, 2009.

[36] R. Rajaram, U. Vaidya, M. Fardad, and B. Ganapathysubramanian, "Stability in the almost everywhere sense: A linear transfer operator approach," J. Math. Anal. Appl., vol. 368, no. 1, pp. 144-156, Aug. 2010.

[37] U. Vaidya and P. G. Mehta, "Lyapunov Measure for Almost Everywhere Stability," IEEE Trans. Automat. Contr., vol. 53, no. 1, pp. 307-323, 2008.

[38] G. Gan, "Effective depth of fresh air distribution in rooms with single-sided natural ventilation," Energy Build., vol. 31, no. 1, pp. 65-73, 2000.

[39] S. C. Hu and C. Y.K., "Deterministic simulation and assessment of airrecirculation performance of unidirectional-flow cleanrooms that incorporate age of air concept," Build. Environ., vol. 38, no. 4, pp. 563-570, 2003.

[40] Z. Shi, J. Chen, and Q. Chen, "On the turbulence models and turbulent Schmidt number in simulating stratified flows," J. Build. Perform. Simul., no. May, 2015.

[41] M. D. Gunzburger, Perspectives in flow control and optimization, Vol. 5. Siam, 
2003.

[42] J. G. Kemeny and J. L. Snell, Finite Markov chains, Vol. 356. Princeton, NJ: van Nostrand, 1960.

[43] S. C. Chapra and R. P. Canale, Numerical Methods for engineers, Vol. 2. McGrawHill, 2012.

[44] M. Budišić, R. Mohr, and I. Mezić, "Applied Koopmanism," Chaos An Interdiscip. J. Nonlinear Sci., vol. 22, no. 4, p. 047510, 2012.

[45] B. Eisenhower, T. Maile, M. Fischer, and I. Mezi, "Decomposing building system model data for model validation and analysis using the Koopman operator," in Fourth National Conference of IBPSA-USA, 2010, no. Jensen 1995.

[46] M. Georgescu, B. Eisenhower, and I. Mezić, "Creating zoning approximations to building energy models using the Koopman operator," IBPSA-USA SimBuild, pp. 40-47, 2012.

[47] A. D. Fontanini, U. Vaidya, and B. Ganapathysubramanian, "A methodology for optimal placement of sensors in enclosed environments: A dynamical systems approach," Build. Environ., vol. 100, pp. 145-161, 2016.

[48] A. D. Fontanini, U. Vaidya, and B. Ganapathysubramanian, "Constructing Markov matrices for real-time transient contaminant transport analysis for indoor environments," Build. Environ., vol. 94, pp. 68-81, 2015.

[49] A. Fontanini, M. G. Olsen, and B. Ganapathysubramanian, "Thermal comparison between ceiling diffusers and fabric ductwork diffusers for green buildings," Energy Build., vol. 43, no. 11, pp. 2973-2987, Nov. 2011.

[50] A. D. Fontanini, A. Passlacqua, U. Vaidya, M. G. Olsen, and B. Ganapathysubramanian, "High-Resolution Performance Analysis of a Large Building with Linear Dispersion Ductwork System," ASHRAE Trans., vol. 120, no. 1, pp. 1-10, 2014.

\section{Appendix A}

In order to test the sensitivity of the Markov matrix to the timestep used to calculate the matrix, three different Markov matrices were calculated with a timestep of 0.1 (sec), 0.5 (sec), and $1.0(\mathrm{sec})$. The mean Courant numbers associated with these timesteps are $0.13,0.64$, and 1.3 respectively. The internal age of air field was sampled along the same locations presented in figure 5, and the results are shown in figure 10. The results show that the Markov method for calculating age of air provides a fairly accurate profile at these locations compared to the experimental values. The results of the two larger timesteps of 0.5 (sec) and $1.0(\mathrm{sec})$ are almost overlaid on top of each other, while the smallest timestep of 0.1 (sec) increases the age of air at these locations. This may be due the small timestep. During the calculation process of the Markov matrix, a small timestep results in the majority of the states having 
large values along the diagonal. These small values on the off diagonal may not fully capture the transition probabilities. Based on these results, it is recommended by the authors that a mean Courant number larger that 0.5 is needed to capture the transition probabilities needed for these calculations.
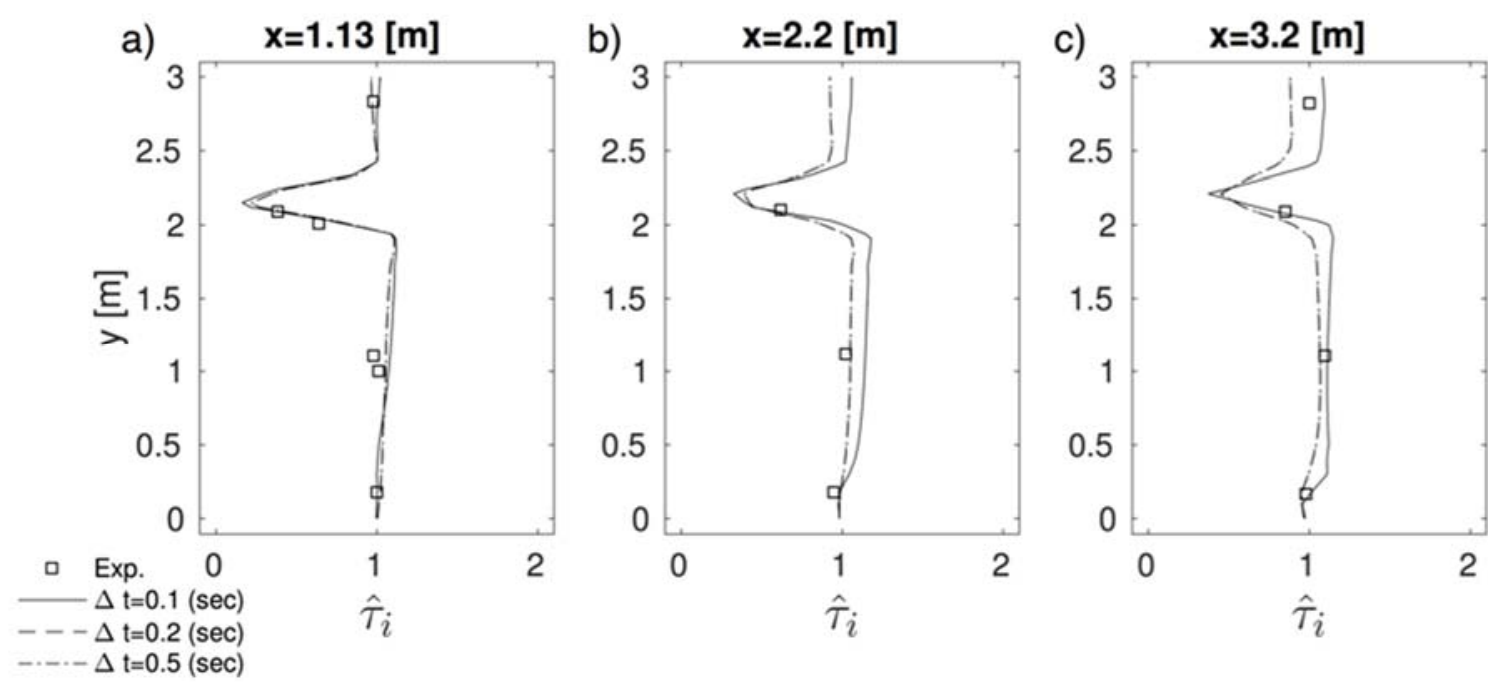

Figure 10: The normalized internal age of air comparisons for different timesteps associated with the Markov matrix. The locations of the are along the z-axis symmetry plane from floor to ceiling at an $x$-location of a) $x=1.13,(\mathrm{~m}) \mathrm{b}) 2.20(\mathrm{~m})$, and c) $3.2(\mathrm{~m})$ 